\title{
Unsteady Gust Response of Road Vehicles
}

\author{
Antonio Filippone \\ UMIST \\ Dept. of Mechanical, Aerospace, Manufacturing Engineering \\ Manchester M60 1QD \\ United Kingdom
}

\begin{abstract}
A theoretical model based on an indicial method is proposed to simulate the unsteady response of a series of road vehicles, including high speed trains, sports utility vehicles, sports cars, caravans, pick-up trucks. The response is described in the frequency domain by the aerodynamic admittance for both side force and yawing moment. The properties of the admittance function are discussed for basic two-dimensional geometries, and the existence of critical damping is shown for a number of cases. The vehicles are undergoing aerodynamic forcing in the form of a gust. Systems with one degree of freedom were considered. The results show that the main parameters affecting the vehicle's aerodynamic response are the mean vehicle length compared to the wave length of the gust, and the inclination of the nose.
\end{abstract}

\section{Introduction}

The aerodynamics of road vehicles is generally unsteady because of the proximity of other vehicles, wind shear, and natural gusts, e.g. cross wind effects, particularly around exposed areas. Simulations of transients and gust conditions are difficult to perform, and reliable experimental data are scarce.

Unsteadiness in the form of lateral gusts is known to affect the handling of some vehicles. Cross winds on high speed trains have become increasingly important, because the speeds have increased, just as the inertial masses have decreased. Strong 
gusts decrease the loading of the wheels on the windward side, which compromises the train's stability at high speeds. A train that encounters a gust at the exit of a tunnel is another critical scenario (Schetz [9]).

Performing laboratory experiments to simulate impulsive side winds requires placing the vehicle in a wind tunnel with a system capable of producing aerodynamic forcing of known characteristics in the transverse direction (frequency, wave length, shape, spectra). A discussion of methods for carrying out these experiments, and their limitations, was published by Bearman and Mullarkey [1].

Problems characterized by reduced frequencies (as defined later) $k>0.2$ must be considered highly unsteady. The study of the unsteady flow occurring at relatively large frequencies can be pursued through the use of the indicial functions. This approach was devised to describe the system's response in the time domain by using an appropriate sequence of step changes in the aerodynamic forcing, and by deriving the corresponding transfer functions. Some of these methods are now quite well established, and applications range from airfoil theory to the fixed and rotary wing (Leishman, 1997 [6]). Their usefulness is particularly exciting when the indicial functions can be expressed in analytical form. In fact, using the Duhamel superposition, solutions can be obtained by straightforward methods, either directly or by numerical integration. More complex forcing functions can be studied using Fourier transforms.

The study of these flows is best performed in the frequency domain, because the power spectra density (PSD) are easily found from the admittance values.

The first mathematical formulations of indicial methods go back to the 1930s: Theodorsen [10] derived expressions for the aerodynamic forces on an oscillating airfoil or airfoil-flap combination with three independent degrees of freedom; Wagner [11] (1925) derived indicial functions for the time-dependent lift of an airfoil subject to impulsive change in the angle of attack. Küssner [5] (1936) modelled the response of an airfoil to a sharp-edged fixed gust. From these basic formulations, extensions were made possible by a number of authors. One case that is necessary to mention is the theory of Drischler and Diederich [2] (1957), which is the starting point of the 
theoretical formulation of the present study. Drischler and Diederich proved that the speed of the travelling sharp-edged gust has a strong effect on the lift and moment response. The system undergoing lateral gusts is obviously forced to accelerate in that direction, at a rate depending on the inertial masses involved (some of these cases were calculated by Drischler and Diederich for a number of wings). This lateral acceleration was not part of the present study.

The paper focusses on two aspects: 1.) On the formulation of suitable transfer functions for the lift/side force and pitching/yawing moment in the frequency domain; 2.) On the application of the theory to road vehicles of arbitrary configuration (in the $\{x, z\}$ plane) undergoing gusts of any frequency. No yawed conditions will be considered (for reasons explained in the theoretical derivation). Two quantities are of interest: the maximum side force and yawing moment response, and the time lag of the response with respect to the forcing function.

The model presented in this paper was published in a detailed form in an earlier study, Filippone-Siquier [3]. Here only the governing equations are given, along with the reference systems and the basic definitions of the main quantities.

\section{Theoretical Model}

The admittance $H$ is the ratio between aerodynamic loads (side force and yawing moment) in unsteady state and the loads created by a forcing of the same amplitude and infinitely large wave length (e.g. quasi steady loads). The relationship between the admittance and the power spectra density,

$$
P S D(k) \sim 2 H^{2}(k)
$$

is relatively simple, since it does not require the Fourier transform of the system's response in the time domain. A suitable form of the transfer function $H(k)$ is the subject of this theory.

The flow model is a two-dimensional incompressible potential flow subject to sufficiently small perturbations. The direction of the normal to the plane flow is affected 
by no separation in the case of lifting surfaces. For the vehicles that were considered for this study, there is a bluff body separation problem. There is a strong limitation only for cases of large yaw angles, as shown later.

Consider the reference system of Fig. 1 in the plane $\{x, z\}$ of the flow, and a downstream travelling gust normal to this plane characterised by a gust speed parameter

$$
\lambda=\frac{V}{V+V_{g}}=\frac{1}{1+V_{g} / V}
$$

In Eq. $2 V$ is the speed of the system and $V_{g}$ is the speed of the gust. The gust speed parameter is singular at $V_{g} / V=-1$. If the gust is convected by the free stream, then $V_{g}=0$, and the gust is said to be "stationary".

In this reference system the aerodynamic admittance of an arbitrary two-dimensional body is found from

$$
H(k)=\frac{1}{h} \int_{D_{z}} r(z) H_{\lambda}[r(z) k] e^{-i \varphi(z) k} d z
$$

where

$$
H_{\lambda}=e^{-i \lambda k}\left\{\left[J_{0}(\lambda k)-i J_{1}(\lambda k)\right] C(k)+\frac{1}{\lambda} i J_{1}(\lambda k)\right\}
$$

is the admittance for the infinite swept lifting surface (e.g. a body of constant length and infinite width). The factor $C(k)$ in Eq. 4 is the Theodorsen function [10]

$$
C(k)=F(k)+i G(k)
$$

with

$$
F(k)=\frac{J_{1}\left(J_{1}+Y_{1}\right)+Y_{1}\left(Y_{1}-J_{0}\right)}{\left(J_{1}+Y_{0}\right)^{2}+\left(Y_{1}-J_{0}\right)^{2}} \quad G(k)=\frac{Y_{1} Y_{0}+J_{1} J_{0}}{\left(J_{1}+Y_{0}\right)^{2}+\left(Y_{1}-J_{0}\right)^{2}}
$$

The quantities $J_{0}, J_{1}, Y_{0}, Y_{1}$ are Bessel functions of the first and second kind in the argument $k$. The expression of these functions is found in many handbooks of mathematics (for example, Press et. al, 1992 [8]). The Theodorsen function can be plotted in several ways; a particularly useful one is the plot of the phase lag $G(k)$ of the airfoil lift against the reduced frequency (Leishman, 2001 [7]). 
Eq. 4 is the basic transfer function for the infinite lifting surface. Eq. 3 is an integral form of $H_{\lambda}(k)$ obtained from the concept of "strip theory" applied to a finite body; $H_{\lambda}(k)$ must be calculated only once, and can be used for an arbitrary body. The strip theory is based on the assumption (now widely accepted) that the downwash velocity at one spanwise location does not affect the downwash at a nearby position. Note that $H_{\lambda}(k)$ is a complex function, fully defined by the magnitude of the response and its time lag with respect to the forcing, just as the Theodorsen's function.

The frequency $\omega$ of the forcing function (in a oscillatory flow) is related to the reduced frequency by

$$
\omega t=\left[\frac{\omega b(z)}{V}\right] s+\left[\frac{\omega f(z)}{V}\right]=\frac{b(z)}{\bar{b}} k s+\frac{f(z)}{\bar{b}} k
$$

where $s$ is the reduced time, $s=V t / b ; b(z)$ is the local length of the body; $f(z)$ is the local position of the leading edge line, Fig. 1. Two functions, appearing in Eq. 3 and Eq. 7 are:

$$
\begin{aligned}
& r(z)=\frac{b(z)}{\bar{b}} \\
& \varphi(z)=\frac{f(z)}{\bar{b}}
\end{aligned}
$$

These definitions can be replaced in Eq. 7. For the reduced frequency one uses the average body length, $\bar{b}$, or the streamwise reference length, $L_{r e f}$.

Eq. 3 shows that at a given frequency the admittance (e.g. the side force and side force coefficient) is inversely proportional to the profile width (or height).

Some general properties of the admittance function are easily found. From Eq. 4 one finds that the limit

$$
\lim _{k \rightarrow 0} H_{\lambda}(k)=1
$$

for all the values of the gust speed ratio $\lambda$. This result is coherent with the definition of admittance as a limit of quasi-steady forcing response. 
For $\lambda=1, \log |H|$ has an asymptote in $-(1 / 2) \log k$ for $k$ tending to infinity. For $\lambda \neq 1, \log \left|H_{k}\right|$ is an oscillating function bounded by two asymptotes whose slope is $\pm 1 / 2$. For $\lambda<1, H_{\lambda}$ has a maximum at the frequency $k \sim 1 / \lambda$. Its magnitude and band-width vary approximately with $1 / \lambda$. For $\lambda<1$ there is no maximum.

\section{$2.1 \quad$ Reduced Frequency}

Some definitions are needed to establish the relationship between the reduced frequency $k$ and the frequency $f$ (in $\mathrm{Hz}$ ), and the relationship between a characteristic length and the wave length of the aerodynamic forcing.

The gust wave length $\lambda_{g}$ is related to its frequency $f$ and the absolute propagation speed by

$$
\lambda_{g}=\frac{V+V_{g}}{f}=\lambda \frac{V}{f}
$$

If $L_{r e f}$ is the reference length of the vehicle, then

$$
\frac{L_{r e f}}{\lambda_{g}}=\frac{L_{r e f}}{V+V_{g}} f=\frac{L_{r e f}}{V+V_{g}} \frac{\omega}{2 \pi}=\frac{k}{2 \pi}
$$

Therefore the ratio between gust wave length and vehicle's reference length is inversely proportional to the reduced frequency; the reduced frequency is proportional to the number of gust wave lengths contained in the reference length:

$$
\frac{\lambda_{g}}{L_{r e f}}=\frac{2 \pi}{k}, \quad k=\frac{L_{r e f}}{\lambda_{g}}
$$

For an airfoil, the characteristic length is the chord. In this case there are at least three ways to define the reduced frequency: One is to use the mean body length, one is to use the maximum length, and finally the body height, $d$. As to the reference speed, it seems appropriate to use the absolute velocity $V+V_{g}$, although $V$ alone could be used. The use of $L_{r e f}=b_{\max }$ does not account for the actual shape of the vehicle, and in this case one could choose the height $d$ instead. When comparing with reference data, the reduced frequency must be defined coherently. Similar incongruence is sometimes found in the definition of the admittance function (some examples are given below). 


\subsection{Admittance for the Moment}

The admittance for the yawing moment around the axis $a$ of a two-dimensional body of length $L_{r e f}=2 b$ and infinite width is found from

$$
H_{\lambda}^{M}(k)=\left(a+\frac{1}{2}\right)\left[J_{0}(\lambda k)-i J_{1}(\lambda k)\right] C(k)-\frac{i}{2 \lambda}(\lambda+2 a) J_{1}(\lambda k)-\frac{1-\lambda}{2 \lambda} J_{2}(\lambda k)(14
$$

where $J_{2}$ is an integer Bessel function of order 2. Eq. 14 can be simplified in at least two cases: If $a=-1 / 2$, then

$$
H_{\lambda}^{M}(k)=\frac{\lambda-1}{2 \lambda}\left[J_{2}(\lambda k)-i J_{1}(\lambda k)\right]
$$

If $\lambda=1$, then

$$
H_{\lambda=1}^{M}(k)=\left(a+\frac{1}{2}\right) H_{\lambda=1}(k)
$$

e.g. the admittance for the yawing moment is proportional to the admittance for the side force, and the two functions are in phase at all frequencies. Normalization of Eq. 16 is done with $a+1 / 2$, so as to have a unit response in the limit of quasi steady flow. This, again, is coherent with the assumption that a very long wave length gust does not affect the steady state loads.

\section{$2.3 \quad$ Effect of Vehicle Length}

The effect of the vehicle length on the aerodynamic admittance can be found in closed form if one considers a rectangle of height $d$ and length $L=A R d$, where $A R$ is the aspect ratio (a factor greater than zero). From Eq. $3 r(z)=1, f(z)=$ constant, $\varphi(z)=$ constant. Therefore:

$$
H(k)=\frac{1}{h} e^{-i \varphi k} \int_{D_{z}} H_{\lambda}(r k) d z=\frac{r}{h} H_{\lambda}(k)
$$

that can be further simplified by taking appropriate coordinate systems. The function $H_{\lambda}$ given by Eq. 4 is not dependent on the geometrical parameter $r$, therefore for given height the admittance of a rectangle is independent of the aspect ratio d/L. 
The leading edge line is invariant to yawing or stretching, therefore it is not possible to simulate yawed conditions. The same conclusion can be reached by looking at the transfer function: this is not dependent on the length of the vehicle. Therefore, the transfer function is invariant to the yaw. This is clearly a drawback, though an obvious one: yawed flaws of bluff bodies feature large areas of flow separation and unsteadiness.

\subsection{Critical Damping}

The critical damping is the opposite of the resonant (or proper) frequency, at which the system's response would be infinite. In a critical damping situation the unsteady forcing creates virtually no loads. The existence of critical damping in this theory arises from the presence of the Bessel functions. The way these operate depends on the integral of Eq. 3.

The admittance for a generic rectangular shape is shown in Fig. 3 for different gust speed ratios. Critical damping occurs at a number of frequencies (generally higher than most practical applications) for positive values of the gust speed ratio, $\lambda>0$. For $\lambda=0$, the unsteady forces may be amplified at some frequencies. Fig. 4 shows the magnitude and the phase lag of $H_{M}(k)$ for the same body. A more interesting analysis would be the phase diagram $\operatorname{Real}\left[H_{\lambda}(k)\right], \operatorname{Imag}\left[H_{\lambda}(k)\right]$ (not shown here for brevity).

Similar critical damping can be found from other simple geometries, such as a triangular shape or a parallelogram of arbitrary inclination. A parallelogram is defined by the geometric parameter $p=\frac{h}{L} \tan \alpha$, where $\alpha$ is the angle with the vertical.

In this case $b(z)=L / 2, r(z)=1, f(z)=z \tan \alpha$. Since $b(z)$ is a constant, a closed form expression for the admittance is found:

$$
H(k)=H_{\lambda}(k) e^{-i p k \lambda} \frac{\sin (p k \lambda)}{p k \lambda}
$$

Critical damping is found at $k_{c}=\frac{n \pi}{p \lambda}$, with $n=1,2,3 \ldots$ The inclination $\alpha$ of the system is the main geometrical parameter with respect to the aerodynamic response to transverse gusts. The larger the parameter $\alpha$, the lower the frequency from where the unsteady response is weak. 
For a circle of radius $R$ centered in $\{R, 0\}$ the relevant quantities in Eq. 3 are:

$$
\bar{b}=\frac{\pi R}{2}, \quad f(z)=R(1-\cos \beta), \quad \varphi(z)=\frac{2(1-\cos \beta)}{\pi}, \quad r(z)=\frac{4 \cos |\beta|}{\pi}
$$

with $\beta=\sin ^{-1}(y / R)$. These parameters introduced in the admittance equations (Eq. 3 and Eq. 4) yield an expression that cannot be solved in closed form, because it involves trigonometric functions in implicit form.

The numerical results, Fig. 5, show that the circle has a number of frequencies with critical damping, the smallest one being $k_{c} \sim 5.13$ at $\lambda=1$. Different critical damping frequencies are found for different gust speed ratios: with $\lambda=0.5$ the smallest critical frequency is $k_{c} \sim 10.38$; no critical frequencies are found with a stationary gust.

\section{Simulation Method}

The basic step for the simulation of generic shape from the governing equations of the present theory to the actual integration, requires the knowledge of the body length $b(z)$ at a given height and the function $\varphi$, as defined by Eq. 9 .

The area $A$ of the arbitrary body is found by a Montecarlo method, which is also straightforward with complex geometries. The body's mean length is $\bar{b}=A / h$. The body is divided numerically into a number $n$ of horizontal strips. The strip length is $b_{i}=A_{i} / d z_{i}$. The function $f(z)$ is found from the position of the leading edge. An example of integration for the SUV is shown in Fig. 2. Fig. 6 shows the admittance for the side force at five gust speed ratios. The result is that the aerodynamic forcing drops at frequencies decreasing with the increasing gust speed ratio.

\subsection{Calculation of Side Force and Yawing Moment}

In Ref. [3] Filippone and Siquier proved that the mean square side force $\overline{C_{S}^{2}}$ is related to the mean square gust angle $\sqrt{\bar{\alpha}^{2}}=\overline{\left(\frac{w}{V}\right)^{2}}$ at a reference frequency $k_{o}$ by 


$$
{\overline{C_{S}}}^{2}=\left[4 \pi^{2} p_{g} \int_{0}^{\infty} \frac{|H(k)|^{2} f\left(k, k_{0}\right)}{\phi(0)} d k\right]{\overline{\left(\frac{w}{V}\right)^{2}}}^{2}
$$

where $|H(k)|$ is the magnitude of the complex number $H(k) ; p_{g}=L_{r e f} / 2 \bar{b}$ is the gust parameter; $f\left(k, k_{o}\right)$ is the function defined by

$$
\begin{array}{r}
f\left(k, k_{0}\right)=\left\{\operatorname{sinc}^{2}\left[p_{g}\left(k_{0}-k\right)\right]+\operatorname{sinc}^{2}\left[p_{g}\left(k_{0}+k\right)\right]-\right. \\
\left.-\cos \left(2 p_{g} k_{0}\right) \operatorname{sinc}\left[p_{g}\left(k_{0}+k\right)\right] \operatorname{sinc}\left[p_{g}\left(k_{0}-k\right)\right]\right\}
\end{array}
$$

In the latter equation $\operatorname{sinc}(x)=\sin (x) / x$ denotes the sine circular function; $f\left(k, k_{o}\right)$ assumes maximum value at $k=k_{o}$, and its oscillations are damped on either side of this frequency, at a rate depending on the gust parameter $p_{g}$. Finally,

$$
\phi(\tau)=\frac{1}{2 T}(T-\tau) \cos \left(\omega_{0} \tau\right)-\frac{1}{2 T}\left(\frac{\sin \left(\omega_{0}(2 T-\tau)\right)-\sin \left(\omega_{0} \tau\right)}{2 \omega_{0}}\right)
$$

is the gust auto-correlation function evaluated at $\tau=0$. In Eq. $21 T$ is the time needed by the gust to travel the reference length with the speed $V$, e.g. $\mathrm{T}=L_{r e f} / V$; $\omega_{o}$ is the frequency associated to $k_{o}$.

The integral term in Eq. 19 depends on the admittance over the whole spectrum of frequencies and on the reference frequency $k_{o}$. Hence $\sqrt{{\overline{C_{S}}}^{2}}$ varies linearly with the root mean square gust angle $\sqrt{\bar{\alpha}^{2}}$. This linear dependence is also valid for the yawing moment coefficient, $C_{Y}$. This coefficient is called $c_{1}$ for the side force and $c_{2}$ for the yawing moment.

Integration of Eq. 19 is carried out as follows:

$$
I=\int_{0}^{\infty} \frac{|H(k)|^{2} f\left(k, k_{0}\right)}{\phi(0)} d k \sim \frac{1}{\phi(0)} \sum_{i=1}^{n}\left|H\left(k_{i}\right)\right|^{2} f\left(k_{i}, k_{o}\right) \Delta k_{i}
$$

with

$$
\phi(0) \sim \frac{1}{2}-\frac{1}{2} \frac{\sin \left(2 \omega_{o} T\right)}{2 \omega_{0} T}
$$

that is a simplification of Eq. 21, and is easily calculated using the sine circular function. In practice, the integral of Eq. 22 converges rapidly. 


\subsection{Comparison with Howell-Everitt's Results}

Howell and Everitt [4] (1983) performed experiments on a 1:25 scale train model mounted on a track in a wind tunnel. This model experienced lateral squared gusts created by centrifugal blowers mounted side by side. The model had two degrees of freedom (in pitch and yaw). Lift and side force admittance were measured.

Using the same two-dimensional shape from a side view, the admittance function for the side force was computed and compared to the wind tunnel data.

The admittance against the reduced frequency used by these authors $k=n d / V=$ $n / 2 g$ was plotted in Fig. $7 ; n$ is the gust frequency (cycles/second), $2 g$ is the gust wave length and $d$ the model width. Also the admittance of a rectangular body whose length is $\bar{b}$ is drawn in Fig. 7 , to show that the vehicle nose has an important role in the determination of its lateral response (a double log scale was used, as in the reference data). It was concluded that the side force has a strong attenuation for a gust wave length about twice the vehicle length (see Eq. 13).

The correlation between wind tunnel and predicted data is satisfactory, although the reference data are not matched exactly. However, the wind tunnel experiments were conducted on models with two degrees of freedom, were affected by the ground proximity (that can be corrected empirically), and were run at a Reynolds number $R e=1.7 \cdot 10^{5}$ (based on the vehicle's width), which is probably around the critical range where the viscous cross-flow effects become substantial.

\subsection{Comparison with Bearman-Mullarkey's Results}

Three of the shapes considered by Bearman and Mullarkey are shown in Fig. 8. These are a family of simplified road vehicles characterized by a back angle "BA" variable between 0 and 40 degrees.

These authors published side force and yawing moment admittance measurements for their BA40 vehicle at yaw angles $\beta$ up to 10 degrees and reduced frequencies

$k=0.54$ and $k=0.99$ for root mean square gust angle of $\sqrt{\bar{\alpha}^{2}}=2.5$ degrees. The data for the side force show little or no variation over the 0 to 5 degrees yaw conditions 
at both frequencies, whilst the admittance for the yawing moment is slightly decreasing over the same range of yaw angles. The present calculations at $\beta=0$, as compared to the experimental data are as follows: $H=0.89$ at $k=0.54$ (wind tunnel: $H=0.87$ ); $H=0.66$ at $k=0.99$ (wind tunnel: $H=0.67$ ). The yawing moment was largely under-estimated at both frequencies. No explanation could be found, except perhaps that the reference point for the yawing moment $(1 / 4$ of the mean length $\bar{b})$ was not the same as in the experimental case.

The fact that values of the forces and yawing moments are constant over a narrow range of yaw angles $(-5<\beta<+5$ degrees) suggests that the three dimensional effects and the effects of bluff body separation can be safely neglected under these conditions.

These authors also show the aerodynamic admittance of side force and yawing moment for the geometry BA0 up to frequencies $k=1.8$ in zero mean yaw angle, $\bar{\beta}=0$. The wind tunnel values are well below unity for $k<1$, suggesting that the normalisation was not done as it was assumed in the present theory (by definition, for $k \rightarrow 1$ the loads tend to the quasi-steady case, and therefore the admittance must have a unit value). Unless we assume that there is an inversion of the data at values $k<0.2$, the results of the present theory must be scaled down to compare with the general trend of the admittance (such an inversion would not be predicted by the theory). The results are shown in Fig. 9, as obtained for a stationary gust, $\lambda=0$ (because the gusts are convected downstream with the free stream velocity) The dip at $k \sim 1$ could not be explained. (No log scale was used in this case, as in the reference data.)

The calculated coefficients in the mean square side force $\overline{C_{S}^{2}}$ vs mean square gust angle (Eq. 19) and mean square yawing moment vs mean square gust angle are summarized in the table below.

\begin{tabular}{c|c|c|c|} 
& BA0 & BA20 & BA40 \\
\hline \hline$c_{1}$ & 0.0392 & 0.0321 & 0.0308 \\
$c_{2}$ & 0.0067 & 0.0059 & 0.0053 \\
\hline
\end{tabular}

Table 1: Calculated side force and yawing moment coefficients.

Using the coefficients in Table 1 for the vehicle BA20, the predicted values of $C_{S}$ 
and $C_{Y}$ have been compared in Fig. 10 with the data of Bearman and Mullarkey.

\section{Results for Road Vehicles}

Different road vehicles were calculated. Fig. 11 shows a comparison of the function $H(k)$ for four different vehicles in the range of reduced frequencies most likely to occur in practice. At a frequency $k=1$ it was found $H \sim 0.698$ for the caravan, $H \sim 0.643$ for the sports utility vehicle, $H \sim 0.549$ for the sports car, and $H \sim 0.396$ for the light pick-up truck. Therefore it appears that at this frequency (as well as lower frequencies), the caravan is the road vehicle subject to the largest unsteady forcing; the light pick-up truck is relatively unaffected by the same aerodynamic forcing, and therefore more stable.

\begin{tabular}{l|c|c|c} 
Vehicle & $\bar{b} / L_{\text {ref }}$ & $H(k=\pi)$ & $k(H=0.5)$ \\
\hline Caravan & 0.844 & 0.466 & 1.321 \\
SUV & 0.758 & 0.356 & 1.213 \\
Sports Car & 0.690 & 0.332 & 1.057 \\
Light Pickup & 0.619 & 0.145 & 0.900 \\
\hline
\end{tabular}

Table 2: Calculated admittance values different road vehicles.

If one defines the cut-off frequency as the reduced frequency at which the system's response decays to a fraction of the quasi steady response (for example, 0.5) then Table 2 shows that: 1.) Cut-off frequencies increase with increasing ratio $\bar{b} / L_{\text {ref }}$ (e.g. the ratio of the mean length over the reference length); 2.) For a given gust whose wave length is twice the reference length (e.g. $k=\pi$, from Eq. 12) the system's response increases with the increasing $\bar{b} / L_{r e f}$.

No experimental data are available to validate the results shown in Fig. 11. One must rely on the comparison with Howell and Everitt's results for the high speed train, to conclude that the results are physically reasonable, and that the most important point is the cut-off length of the vehicle for a given gust wave length. 


\section{Conclusions}

The theoretical model for the aerodynamic admittance of side force and yawing moment has been developed from an extension of the theory of Drischler and Diederich, based on the assumption of two dimensional and small perturbation flow. The theory can be applied to the study in the frequency domain of the unsteady response of a three dimensional body at small yaw angles.

The analysis has shown that for simple two-dimensional shapes there is a large number of frequencies yielding critical damping. This is associated to the oscillatory behaviour of the Bessel functions in the transfer function.

The response to sinusoidal forcing of arbitrary frequency by gusts in a wide range of speeds has been calculated for a number of road vehicles. It is shown that the forcing generally decreases with the increasing frequency, with a rate depending essentially on the mean vehicle's length. Similar results have been obtained for a high speed train, in the simplifying assumption of one degree of freedom. Comparison with the experimental results available were good, even with all the approximations of the case. The model can be further extended as to include two degrees of freedom, arbitrary gusts, and load and stability effects on the vehicle.

However, reliable experimental data in the frequency domain would be needed for a complete validation of the model.

\section{References}

[1] Bearman, PW and Mullarkey, SP. Aerodynamic forces on road vehicles due to steady side winds and gusts. In Road Vehicle Aerodynamics, Royal Aeronautical Society Conference, Loughborough University, UK, 1994, pp. 4.1-4.12.

[2] Drischler, JA and Diederich, FW. Lift and moment responses to penetration of sharp-edged travelling vertical gusts, with application to penetration of weak blast waves. NACA TN 3956, May 1957. 
[3] Filippone, A and Siquier, J. Aerodynamic admittance of two-dimensional bodies. J. Royal Aero. Society (to appear), 2003.

[4] Howell, JP and Everitt, KW. Gust response of a high speed train model. In Annual Meeting of the ASME - Aerodynamics of Transportation (Morel, T. and Miller, J. editors), Vol. 7, pp. 81-89, Boston, MA, Nov. 1983.

[5] Küssner, HG. Zusammenfassender bericht über den instationären auftreib von flügeln. Luftfahrtforschung, 13(12), pp. 410-424, Dec. 1936.

[6] Leishman, JG. Unsteady aerodynamics of airfoils encountering traveling gusts and vortices. J. of Aircraft, 34(6), pp. 719-729, Dec. 1997.

[7] Leishman, JG. Principles of Helicopter Aerodynamics. Cambridge Aerospace Series. Cambridge University Press, 2000.

[8] Press, WH et al. Numerical Recipes. Cambridge Univ Press, 1992. (Chapter 6).

[9] Schetz, JA. Aerodynamics of high-speed trains. Annu. Rev. Fluid Mech., 33, pp. 371-414, 2001.

[10] Theodorsen, T. General theory of aerodynamic instability and the mechanism of flutter. NACA Report 496, 1935.

[11] H Wagner. Über die Entstahung des dynamischen Auftriebes von Tragflügeln. Zeitschrift für Angewandte Mathematik und Mechanik, 5(1), pp. 17-35, Feb. 1925. 


\section{List of symbols}

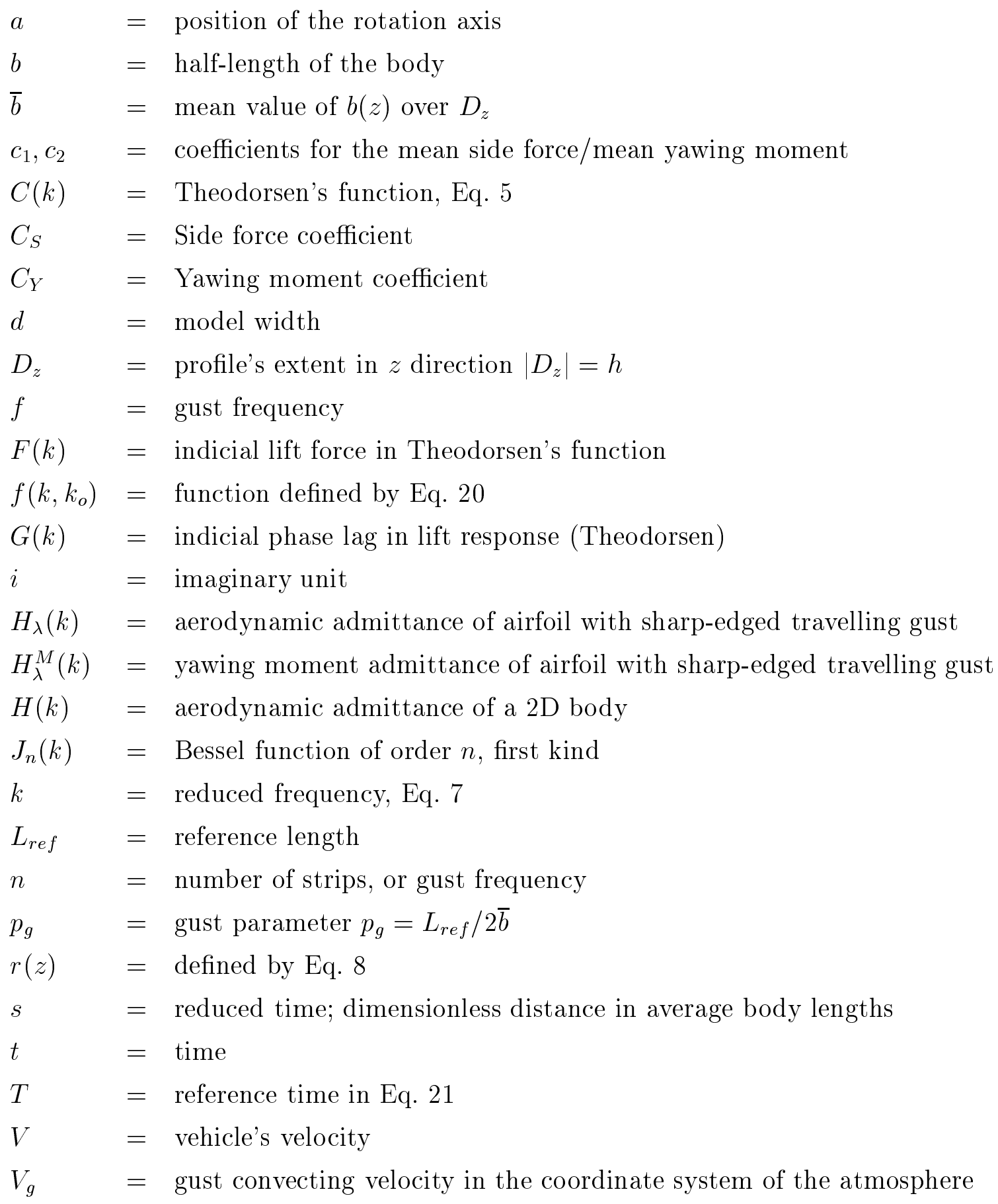




\section{Greek Symbols}

$\alpha=$ angle with the vertical direction of the vehicle's nose; gust angle

$\beta=$ yaw angle

$\lambda=$ gust speed parameter, Eq. 2

$\lambda_{g} \quad=$ gust wave length

$\phi(s)=$ gust auto correlation function

$\varphi(z)=$ defined by Eq. 9

$\omega=$ pulsation in a harmonic movement

\section{Underscripts/Overscripts}

()$_{g}=$ gust

()$_{\lambda}=$ gust speed ratio $\lambda$ as a parameter

$\overline{()^{2}}=$ mean square value over full oscillation

()$_{c}=$ critical value 


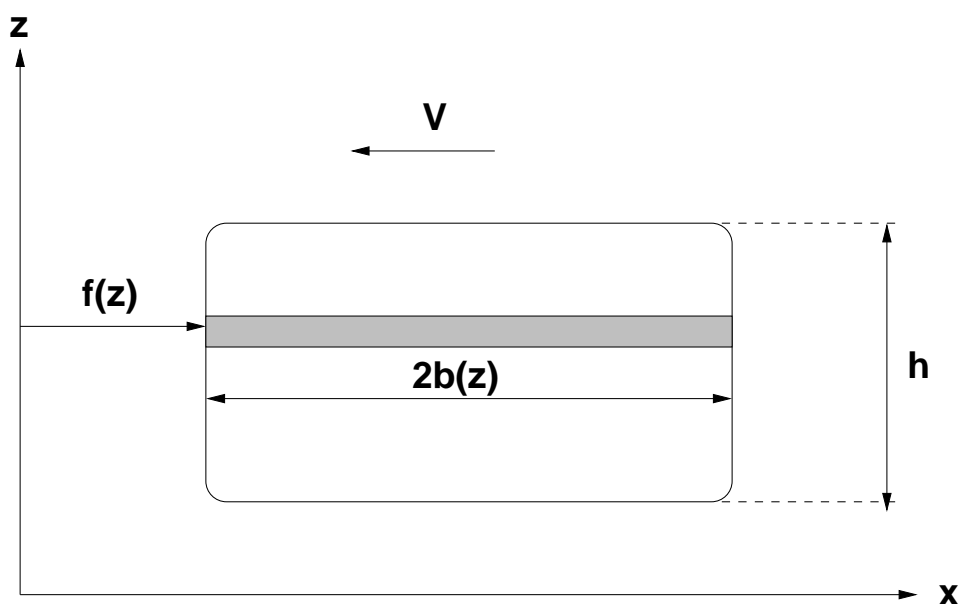

h

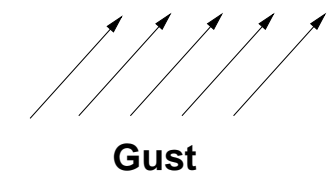

Figure 1: Reference system. 

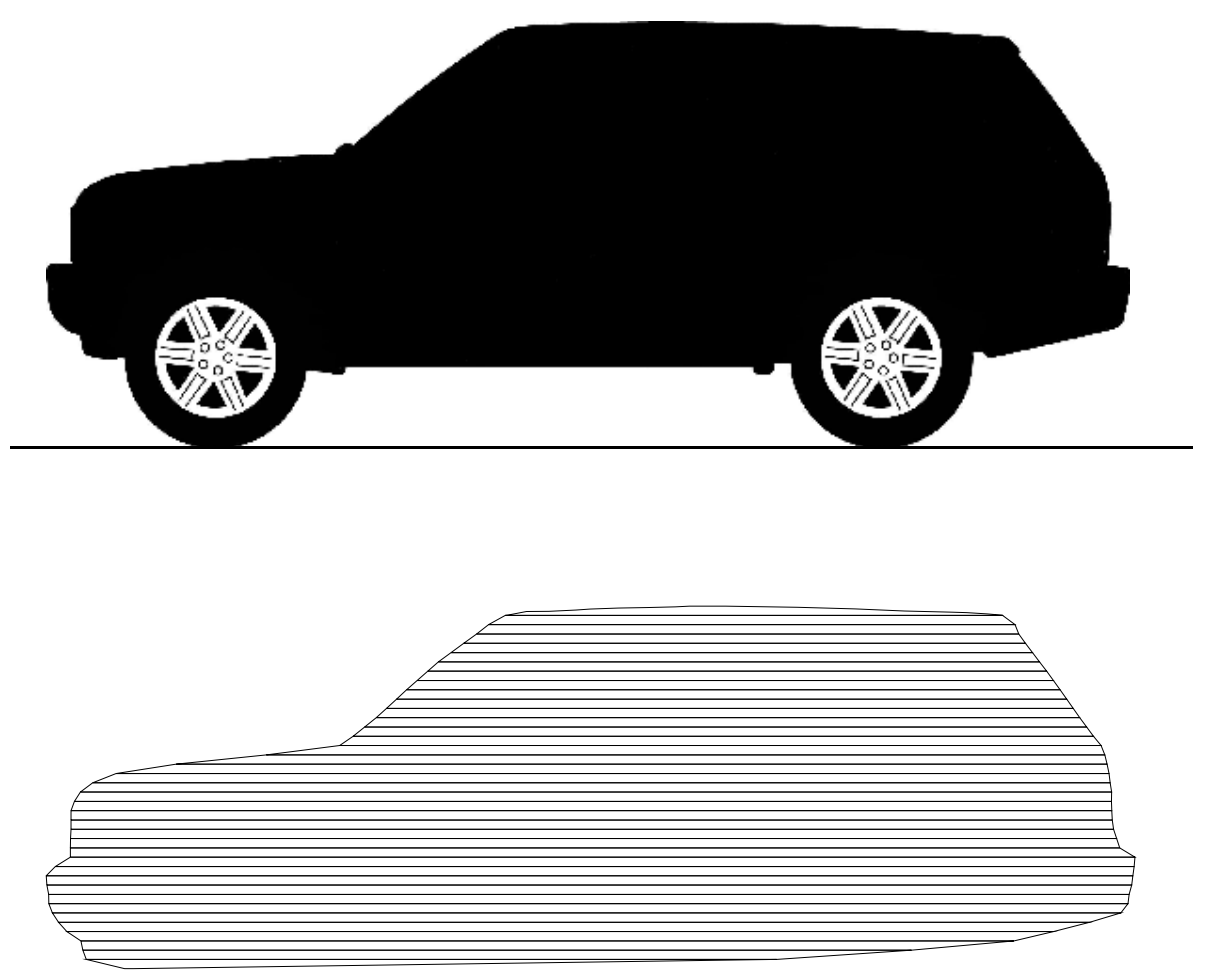

Figure 2: Side view of SUV and computational model (without wheels). 


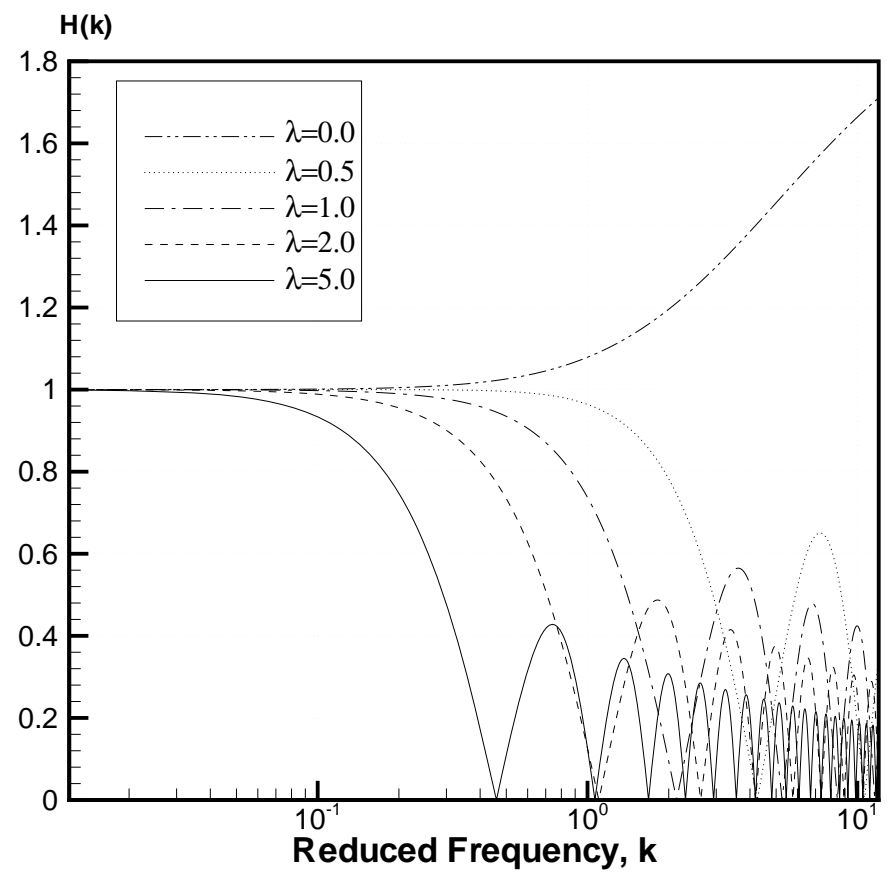

Figure 3: Computed aerodynamic admittance (side force) for a rectangular body for different gust speed ratios (as indicated). 


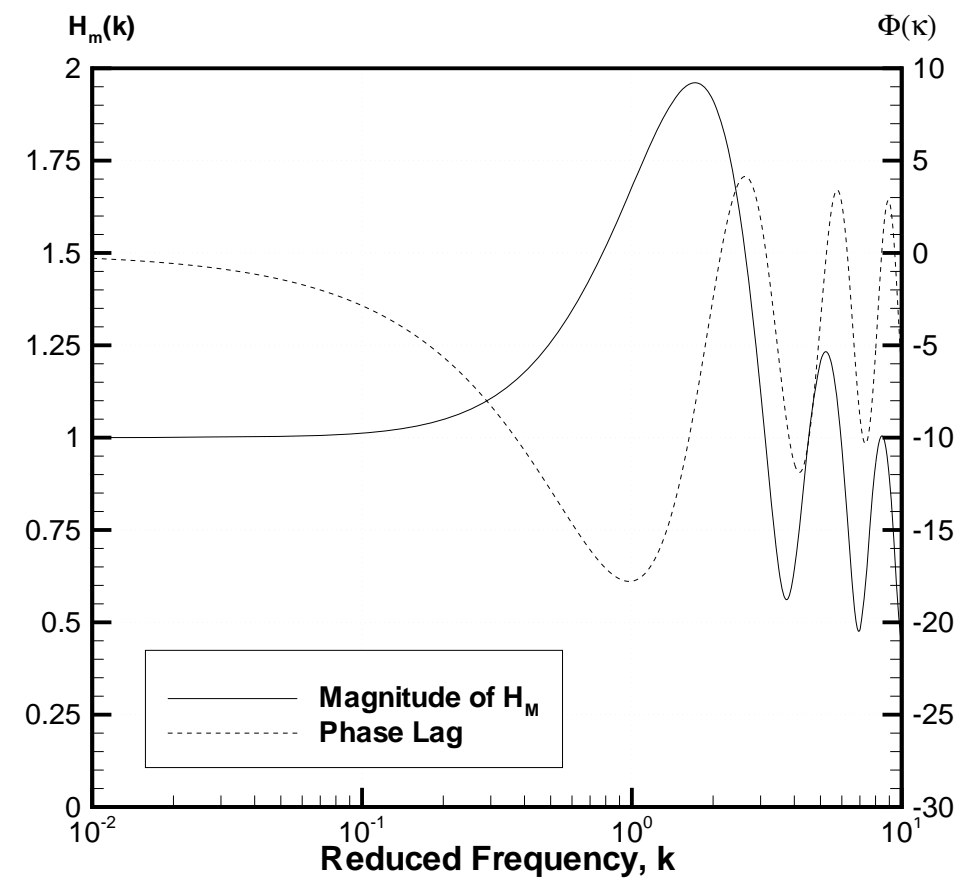

Figure 4: magnitude and phase lag of the yawing moment admittance for a rectangular body, $\lambda=1, a=1 / 4$. 


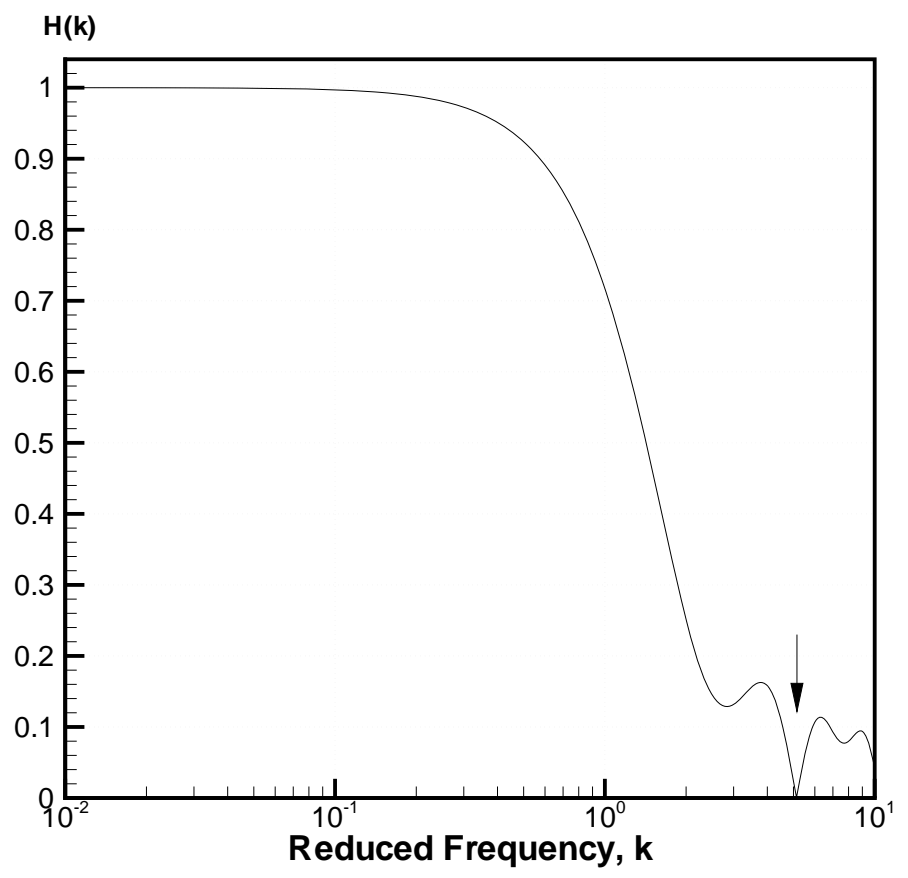

Figure 5: Admittance for a circle, $\lambda=1$, critical frequency $k=5.13$. 


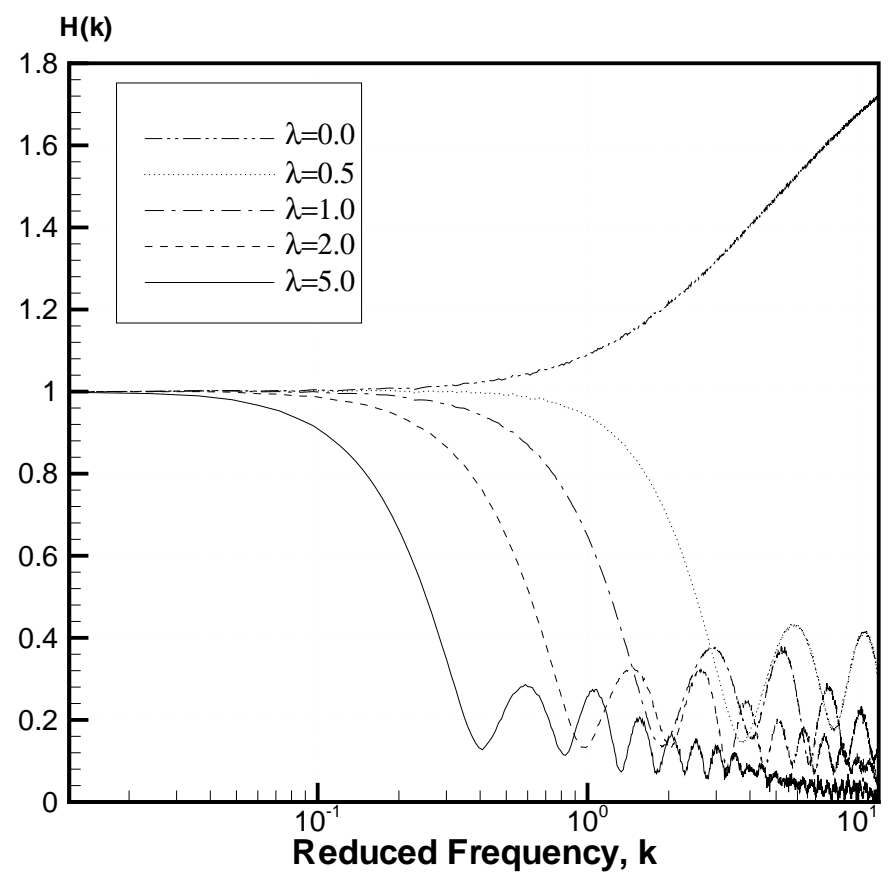

Figure 6: Computed aerodynamic admittance for the model of Fig. 2 for different gust speed ratios (as indicated). 


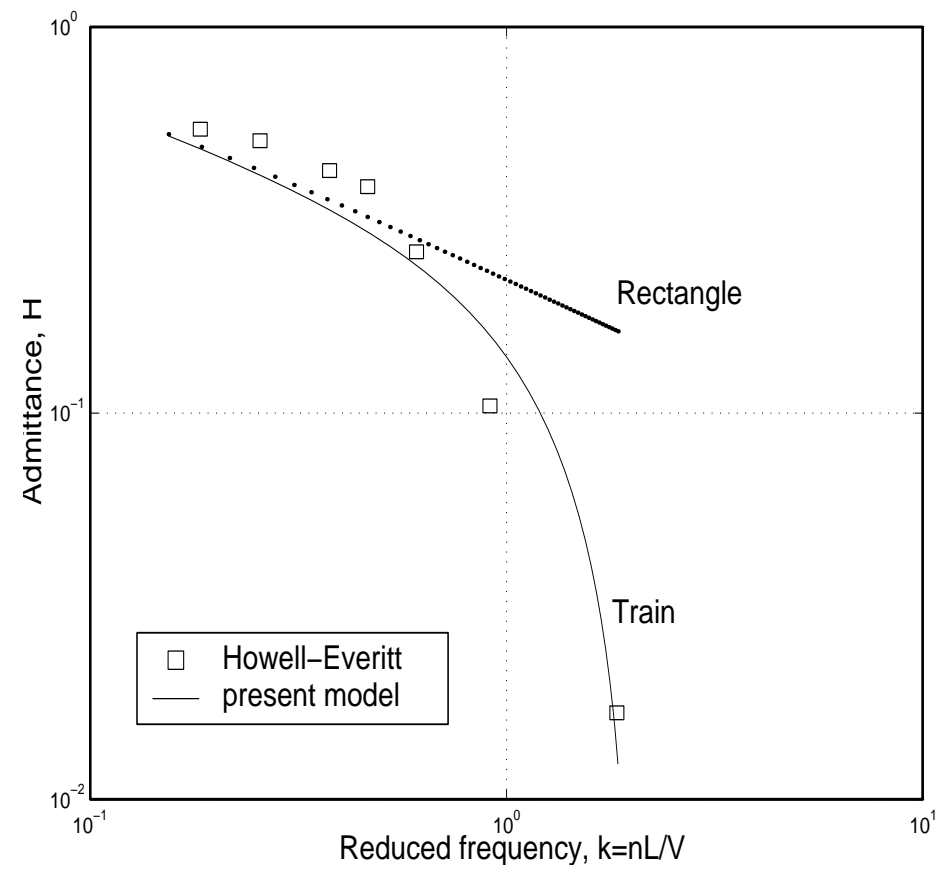

Figure 7: Comparison with Howell and Everitt's data (in double logarithmic scale), Ref. [4]. 


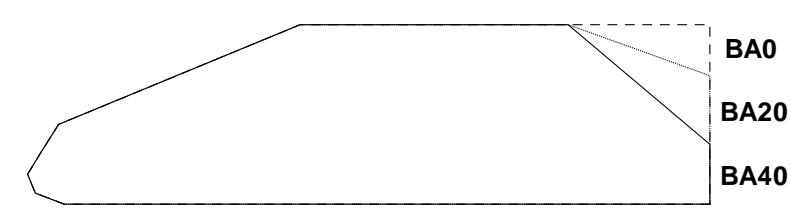

Figure 8: Vehicles BA0, BA20, BA40 considered by Bearman and Mullarkey [1]. 


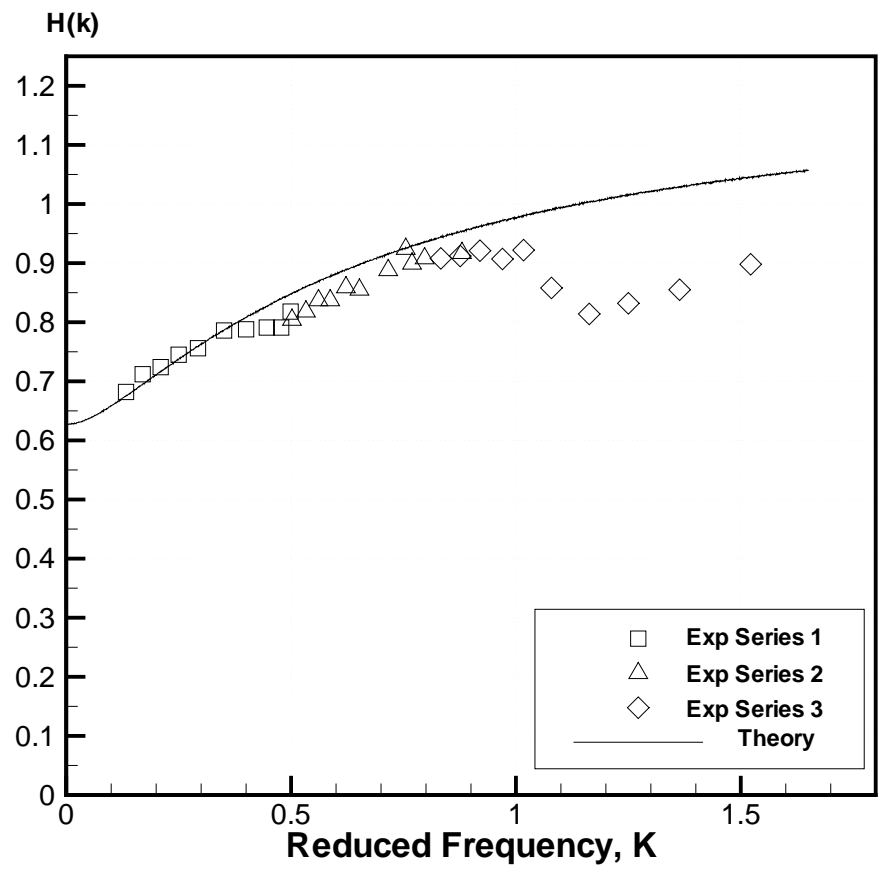

Figure 9: Admittance for the side force of vehicle $B A 0$, set at $\bar{\beta}=0$, with a stationary gust, $\lambda=0$, compared with the experimental data of Ref. [1]. 


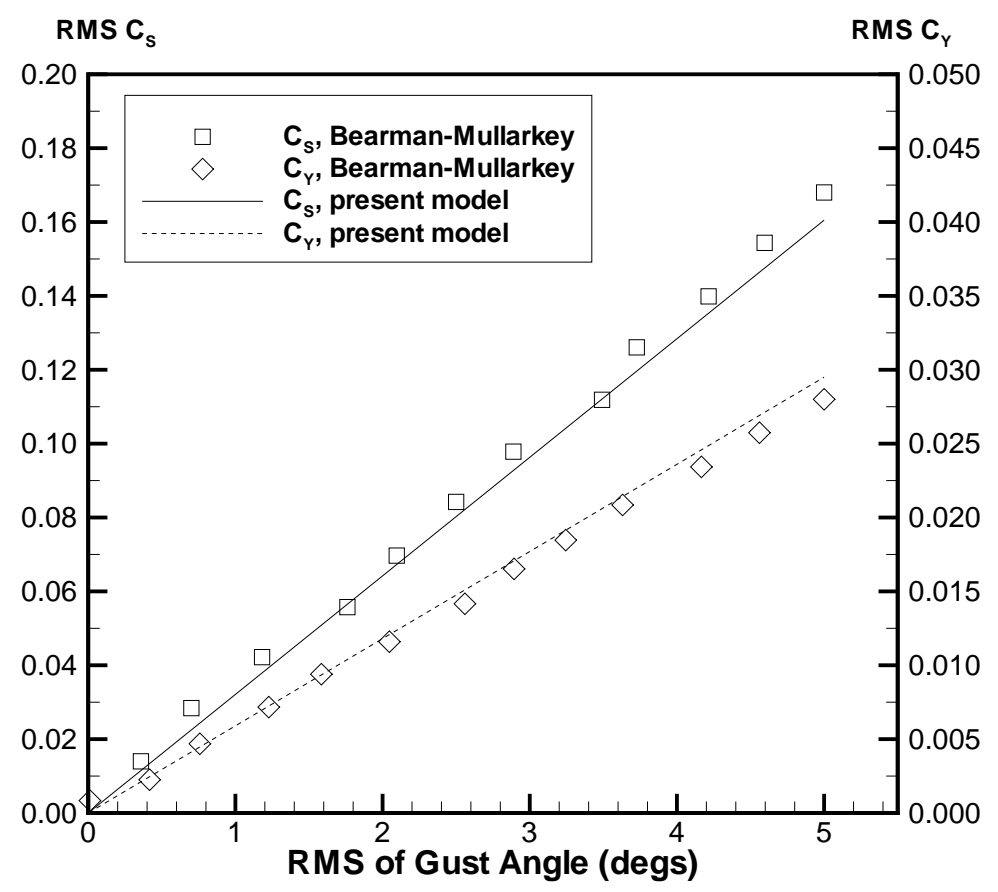

Figure 10: RMS of side force and yawing moment for $\sqrt{\bar{\alpha}^{2}}$ compared with the experimental data of Ref. [1]. 


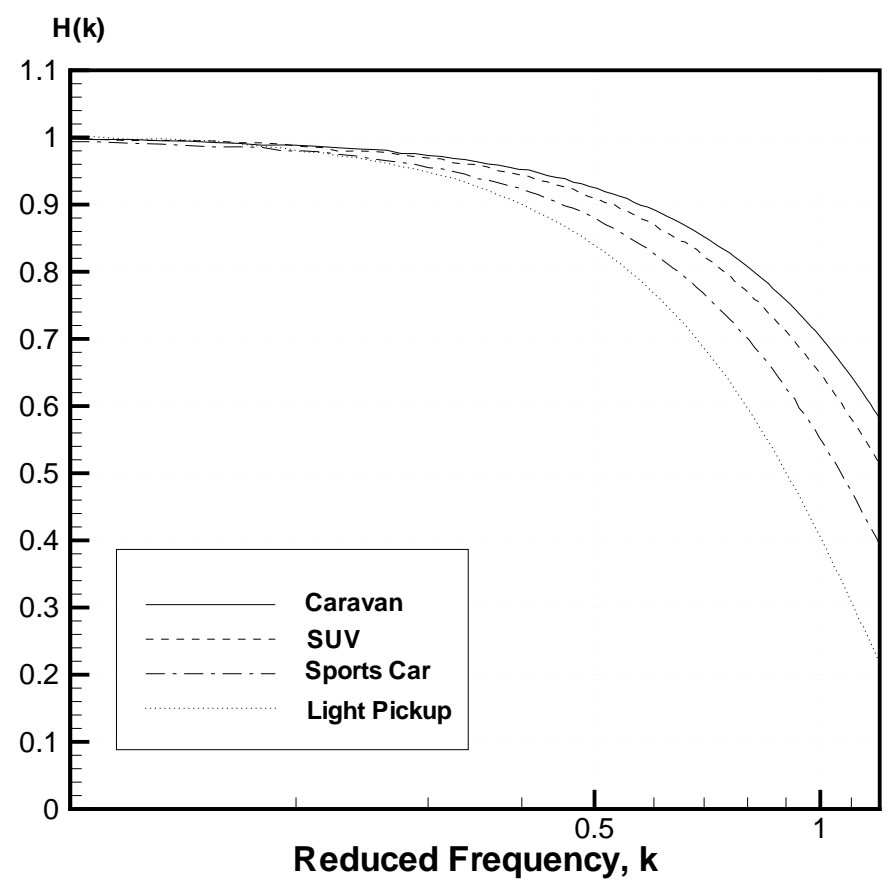

Figure 11: Admittance for 4 different vehicles, for the gust speed ratio $\lambda=1$. 